\title{
Delivering a Practical Framework for Ethical Decision-Making Involving Big Data in Health and Research
}

\author{
Graeme T. Laurie ${ }^{1} \cdot$ E Shyong Tai $^{2,3} \cdot$ on behalf of the SHAPES Working Group
}

Published online: 30 September 2019

(C) National University of Singapore and Springer Nature Singapore Pte Ltd. 2019

The Science, Health and Policy-relevant Ethics in Singapore (SHAPES) Initiative is a programme at the Centre for Biomedical Ethics, National University of Singapore funded by Singapore's National Medical Research Council. SHAPES was funded to develop a Big Data Project in 2018-2019 and established an international Working Group to this end in 2018. This Special Issue presents the Ethics Framework for Big Data in Health and Research ('the Framework') (Xafis et al. 2019) developed by the SHAPES Working Group.

The topic of big data has been explored extensively in academic, technical, government, legal and private sector literature for a number of decades. The potential arising from the use of big data in health and research is widely recognised, as are the challenges posed in this fast-paced dynamic field. However, despite the wealth of literature, there is a lack of practical guidance for decision-making in the form of a framework that considers the ethical issues that arise from the use of big data in a variety of health and research contexts.

The Framework addresses this gap and is intended for a wide-range of professional audiences including biomedical researchers, clinician-researchers, data scientists, policymakers, those involved in the governance of big data activities in health and research (including ethics committees and data access committees) and data controllers. Beyond the professionals who may find this resource helpful, the Framework may also be useful to lay people with an interest in big data, patients and research participants.

The Framework itself comprises three main components: (1) a list of 16 values identified by the Working Group to be central to a number of big data contexts in which

Graeme T. Laurie

Graeme.Laurie@ed.ac.uk

1 School of Law and JK Mason Institute for Medicine, Life Sciences and the Law, University of Edinburgh, Edinburgh, UK

2 Division of Endocrinology, National University Hospital, Singapore

3 Saw Swee Hock School of Public Health, National University of Singapore, Singapore 
Framework users are most likely to find themselves when making decisions, (2) a sixstep deliberative decision-making process to help guide users' thinking during the decision-making process and (3) articulation of three broader considerations that underlie decisions made using the Framework: the need to respect persons, to take account of community expectations and to consider issues of vulnerability, which can arise in uses of big data.

To illustrate how the Framework can be used in practice, it has been applied to six specific contexts. These are published as separate papers and referred to as 'Domains': Openness in big data and data repositories (Xafis and Labude 2019); Precision medicine and big data (Schaefer et al. 2019); The use of real-world data to generate evidence about healthcare interventions (Lipworth 2019); AI-assisted decision making in healthcare (Lysaght et al. 2019); Big Data and Public-Private Partnerships in Healthcare and Research (Ballantyne and Stewart 2019); and Cross-sectoral big data (Laurie 2019). These papers describe some of the context-specific ethical issues and values that are relevant to each Domain. Each Domain then provides examples and a case study to exemplify the workings of the Framework and to demonstrate how we can robustly arrive at and justify decisions where there is more than one 'right' approach.

The Framework, like all decision-making frameworks, does not therefore present solutions to ethical issues raised. Rather, it provides insight into how underlying values ought to occupy a central position in the decision-making process and how values can be balanced to reach justifiable decisions. The Framework differs from other decisionmaking tools as it articulates specific values and provides extensive analyses demonstrating how values and relevant issues are to be considered and thought through. This is the strength of this Framework, as it can function as a practical tool for those working in numerous fields employing big data. The variety and number of cases and examples considered in the Domains provide a model for users and offer ample opportunity to draw on the analyses provided when making decisions.

\section{SHAPES Working Group}

Co-chairs:

Associate Professor Tai E Shyong

Professor Graeme T. Laurie
Division of Endocrinology, National University Hospital; and Saw Swee Hock School of Public Health, National University of Singapore

School of Law and JK Mason Institute for Medicine, Life Sciences and the Law, University of Edinburgh

Members in alphabetical order:

Associate Professor Angela Ballantyne

Dr Iain Brassington

Mr Markus K. Labude

Associate Professor Hannah Yeefen Lim

Associate Professor Wendy Lipworth

Assistant Professor Tamra Lysaght
Department of Primary Health Care \& General Practice, University of Otago

Centre for Social Ethics and Policy, School of Law, University of Manchester

Centre for Biomedical Ethics, National University of Singapore

Division of Business Law, College of Business, Nanyang Technological University

Sydney Health Ethics, The University of Sydney

Centre for Biomedical Ethics, National University of Singapore 


\begin{tabular}{ll}
\hline Dr G. Owen Schaefer & Centre for Biomedical Ethics, National University of Singapore \\
Professor Cameron Stewart & Sydney Law School, The University of Sydney \\
$\begin{array}{c}\text { Associate Professor Shirley } \\
\text { Sun }\end{array}$ & $\begin{array}{r}\text { School of Social Sciences, College of Humanities, Arts, \& Social Sciences, } \\
\text { Nanyang Technological University }\end{array}$ \\
Dr Vicki Xafis & Centre for Biomedical Ethics, National University of Singapore \\
\hline
\end{tabular}

Funding The development of the Framework and its application to the six Domain papers was funded and supported by the Singapore National Medical Research Council Research, Innovation and Enterprise 2020 Grant.

\section{References}

Ballantyne, Angela, and Cameron Stewart. 2019. Big data and public-private partnerships in healthcare and research: The application of an ethics framework for big data in health and research. Asian Bioethics Review 11 (3). https://doi.org/10.1007/s41649-019-00100-7.

Laurie, Graeme T. 2019. Cross-sectoral big data: The application of an ethics framework for big data in health and research. Asian Bioethics Review 11 (3). https://doi.org/10.1007/s41649-019-00093-3.

Lipworth, Wendy. 2019. Real-world data to generate evidence about healthcare interventions: The application of an ethics framework for big data in health and research. Asian Bioethics Review 11 (3). https://doi. org/10.1007/s41649-019-00095-1.

Lysaght, Tamra, Hannah Yeefen Lim, Vicki Xafis, and Kee Yuan Ngiam. 2019. AI-assisted decision-making in healthcare: The application of an ethics framework for big data in health and research. Asian Bioethics Review 11 (3). https://doi.org/10.1007/s41649-019-00096-0.

Schaefer, G. Owen, E Shyong Tai, and Shirley Sun. 2019. Precision medicine and big data: The application of an ethics framework for big data in health and research. Asian Bioethics Review 11 (3). https://doi. org/10.1007/s41649-019-00094-2.

Xafis, Vicki, and Markus K. Labude. 2019. Openness in big data and data repositories: The application of an ethics framework for big data in health and research. Asian Bioethics Review 11 (3). https://doi. org/10.1007/s41649-019-00097-z.

Xafis, Vicki, G. Owen Schaefer, Markus K. Labude, Iain Brassington, Angela Ballantyne, Hannah Yeefen Lim, Wendy Lipworth, Tamra Lysaght, Cameron Stewart, Shirley Sun, Graeme T. Laurie, and E Shyong Tai. 2019. An ethics framework for big data in health and research. Asian Bioethics Review 11 (3). https://doi.org/10.1007/s41649-019-00099-x.

Publisher's Note Springer Nature remains neutral with regard to jurisdictional claims in published maps and institutional affiliations. 